representatives of the principal economic Powers in particular, Great Britain, the United States, France, Germany, and Italy-in the hope that they may succeed in working out an agreed plan of international economic collaboration. That there is ground for this hope is clear from the fact that everywhere he went M. Van Zeeland tells us that he met with a most sympathetic reception. The report he has so carefully prepared should provide an excellent basis for discussion. It is well balanced, stating frankly the difficulties which have to be faced without being too caustically critical of the narrow nationalistic aims recently pursued by nearly all countries.

Among the more fundamental problems for which a solution must be found, two in particular have received a good deal : of advertisement, different countries advocating claims and counter-claims in regard to them, namely, those which relate to raw materials and colonies. It is highly important that such problems should be approached, as M. Van Zeeland urges in his report, "in a spirit of complete objectivity". That is a sound principle which every man of science will be ready to endorse. As to colonies, the most promising line of attack seems to be, again quoting the words of the report, "to seek for the means of generalizing the system of the open door which obtains in the Conventional Basin of the Congo, a system the general result of which it is impossible to criticize". So long as colonial Powers are in a position to exercise monopoly rights in the areas they control, dissatisfaction is bound to remain in the minds of those ruling the nations that are shut out, and such dissatisfaction is the potential seed of war.

\section{Niagara Falls Bridge Disaster}

The well-known Niagara Falls View Bridge across the Niagara River, just below the falls, a steelframed structure in a single span of about 1,200 feet, weighing some 2,600 tons and erected in 1898 at a cost of 160,000 , collapsed during the afternoon of January 27, under the pressure exerted by an enormous mass of pack ice which had accumulated at the foot of the Falls. By reason of the intense cold of the preceding weeks, the ice had piled itself in huge blocks as it was swept over the Falls, to a height not much less than the deck level of the bridge, which was 165 feet above the river, and the danger had been clearly recognized for some time previously, so that when, despite the efforts made to save the bridge with heavy timber fenders, the final collapse took place, the sight was witnessed by a crowd estimated at ten thousand. The broken bridge now rests in a tangle of twisted steelwork upon the surface of the ice, which continues to gather and fills the gorge for a distance of two or three miles. There is another bridge for rail and road traffic about a mile and a half downstream, and this will have to be utilized for pedestrians until the fallen bridge is replaced. The drift of ice towards Lake Ontario is causing concern lest damage should be done to the generating station and plant of the Ontario Hydro-electric
Commission, which is a short distance below the Falls. As it is, there is serious interference with the supply of electricity due to the fracture of mains.

\section{Decibels and Phons}

A CORRESPONDENT has raised the question of the use of the decibel as a unit for expressing sound intensity. Since his difficulties are shared by others, it seemed worth while to place them before an authority on the subject, who has been good enough to deal with them. He points out that it is not always realized that the decibel is a unit for specifying changes in intensity, power, or energy, and not a direct measure of the absolute value of these quantities. Decibels are not additive, since they measure changes on a logarithmic scale; so that it is the ratio of two sound intensities which determines their difference in decibels. Two similar sounds of intensities $I$ and $I_{0}$ are said to differ in intensity by $n$ decibels when $n=10 \log _{10} I / I_{0}$. Hence a 10 -fold increase in the intensity of a sound is a 10 decibel change, a 100-fold increase a 20 decibel change, and a 1000-fold increase a 30 decibel change. A decibel corresponds to an increase of intensity or energy in a ratio of approximately $5 / 4$, two decibels to a ratio of $(5 / 4)^{2}$, three to a ratio of $(5 / 4)^{3}=2, \ldots$ and so on. Thus doubling the intensity of a sound, as when two similar voices sing the same note, corresponds to a change of 3 decibels. When a sound is said to have an intensity of so many decibels, it is implied that the intensity is being compared with some intensity which has been selected as a zero level. An international conference held in Paris last July adopted an intensity corresponding to an acoustical pressure of 0.0002 dyne per sq. $\mathrm{cm}$. as the reference zero, this being near the threshold of hearing for sounds with frequencies in the neighbourhood of 1000 cycles per second.

Ir will be seen that the decibel is a purely physical unit and is independent of the characteristics of the ear, which assesses loudness and not intensity. Moreover, two sounds of the same intensity but of different quality will not necessarily appear to be equally loud. For comparing the loudness of sounds, a scale of phons has been set up, based upon the fact that individuals find it possible to judge whether sounds of differing quality produce a sensation of equal loudness. Any sound or noise is matched under specified conditions against a pure reference tone with a frequency of 1000 cycles per second. If the intensity of the reference tone in decibels above the zero level is $n$, the reference tone and the sound or noise are said to have an equivalent loudness of $n$ phons. For sounds of medium frequency and moderate loudness, a phon happens to correspond roughly to the smallest difference of loudness which can be detected by alternate listening under ordinary conditions.

\section{Educational Science at Cambridge}

THE recommendations of the Special Syndicate at the University of Cambridge on the position of the 